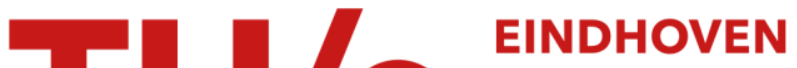 UNIVERSITY OF TECHNOLOGY
}

\section{Magnetic field effects on tactoids of plate-like colloids}

\section{Citation for published version (APA):}

Verhoeff, A. A., Otten, R. H. J., Schoot, van der, P., \& Lekkerkerker, H. N. W. (2011). Magnetic field effects on tactoids of plate-like colloids. Journal of Chemical Physics, 134(4), 044904-1/14. [044904].

https://doi.org/10.1063/1.3520389

DOI:

10.1063/1.3520389

Document status and date:

Published: 01/01/2011

\section{Document Version:}

Publisher's PDF, also known as Version of Record (includes final page, issue and volume numbers)

\section{Please check the document version of this publication:}

- A submitted manuscript is the version of the article upon submission and before peer-review. There can be important differences between the submitted version and the official published version of record. People interested in the research are advised to contact the author for the final version of the publication, or visit the $\mathrm{DOI}$ to the publisher's website.

- The final author version and the galley proof are versions of the publication after peer review.

- The final published version features the final layout of the paper including the volume, issue and page numbers.

Link to publication

\section{General rights}

Copyright and moral rights for the publications made accessible in the public portal are retained by the authors and/or other copyright owners and it is a condition of accessing publications that users recognise and abide by the legal requirements associated with these rights.

- Users may download and print one copy of any publication from the public portal for the purpose of private study or research.

- You may not further distribute the material or use it for any profit-making activity or commercial gain

- You may freely distribute the URL identifying the publication in the public portal.

If the publication is distributed under the terms of Article 25fa of the Dutch Copyright Act, indicated by the "Taverne" license above, please follow below link for the End User Agreement:

www.tue.nl/taverne

Take down policy

If you believe that this document breaches copyright please contact us at:

openaccess@tue.nl

providing details and we will investigate your claim. 


\title{
Magnetic field effects on tactoids of plate-like colloids
}

\author{
A. A. Verhoeff,, a) R. H. J. Otten, ${ }^{2}$ Paul van der Schoot, ${ }^{3}$ and H. N. W. Lekkerkerker ${ }^{1}$ \\ ${ }^{1}$ Van 't Hoff Laboratory for Physical and Colloid Chemistry, Debye Institute for NanoMaterials Science, \\ Utrecht University, Padualaan 8, 3584 CH Utrecht, The Netherlands \\ ${ }^{2}$ Group Theory of Polymers and Soft Matter, Eindhoven Polymer Laboratories, Eindhoven University of \\ Technology, P.O. Box 513, 5600 MB Eindhoven, The Netherlands, and Dutch Polymer Institute, P.O. Box 902, \\ 5600 AX Eindhoven, The Netherlands \\ ${ }^{3}$ Group Theory of Polymers and Soft Matter, Eindhoven University of Technology, P.O. Box 513, 5600 MB \\ Eindhoven, The Netherlands, and Institute for Theoretical Physics, Utrecht University, Leuvenlaan 4, \\ 3584 CE Utrecht, The Netherlands
}

(Received 17 September 2010; accepted 4 November 2010; published online 26 January 2011)

\begin{abstract}
We investigate the effect of a magnetic field on the shape and director field of nematic droplets in dispersions of sterically stabilized and charge-stabilized colloidal gibbsite platelets with a negative diamagnetic anisotropy. Depending on the magnetic field strength and tactoid size, we observe with polarized light microscopy several interesting structures, with different shapes and director fields both with and without defects. In particular, our findings provide the first experimental evidence for the existence of the split-core defect structure predicted ten years ago by Mkaddem and Gartland [Phys. Rev. E 62, 6694 (2000)]. The split-core structure is a metastable director-field configuration that can be stabilized by a sufficiently strong externally applied magnetic field but only if the diamagnetic anisotropy of the particles is negative. To account for our observations, we present a calculation of the stability regions of different shapes and director-field structures as a function of tactoid size, anchoring conditions, surface tension, elastic constants, and magnetic field strength. By fitting the experimental data to the theoretically predicted structures, we are able to extract values for the splay elastic constant, interfacial tension, and anchoring strength. Remarkably, we find significant differences between the two systems studied: for sterically stabilized gibbsite in bromotoluene the anchoring strength is one order of magnitude larger than that of aqueous gibbsite, with the latter exhibiting weak and the former strong anchoring of the director field to the interface. The splay elastic constants that we obtain are in agreement with earlier experiments, simulations, and theory, while the interfacial tension and anchoring strength are considerably larger than what was found in earlier experiments. @ 2011 American Institute of Physics. [doi:10.1063/1.3520389]
\end{abstract}

\section{INTRODUCTION}

Tactoids are nematic droplets formed in the early stages of the emergence of a nematic liquid-crystalline phase in suspensions of sufficiently anisometric colloidal particles. They are an interesting object of study because their shape and director-field configuration are determined by a competition between elastic and interfacial forces, the balance of which is a function of their size. ${ }^{1-3}$ This implies that the anchoring strength, surface tension, and elastic constants can, at least in principle, be deduced from the size dependence of the shape and internal structure of tactoids.

Tactoids formed in suspensions of "elongated" (rod-like) colloidal particles have been studied for quite some time in a variety of systems, ranging from suspensions of vanadium pentoxide, ${ }^{4,5}$ tobacco mosaic virus, ${ }^{6}$ and fd virus, ${ }^{7}$ to F-actin. ${ }^{8}$ Very recently, tactoids in systems of rods have also been investigated by means of computer simulation. ${ }^{9-12}$ These nematic droplets have an unusual, elongated (spindle-like) shape and are characterized by a bipolar director field as is evidenced by polarization microscopy, a consequence of the

\footnotetext{
a)Electronic mail: a.a.verhoeff@uu.nl.
}

planar anchoring of the nematic director field to the isotropicnematic interface observed in this kind of system., ${ }^{53-15}$

By fitting theoretical predictions to shape characteristics such as the aspect ratio and the tip angle for tactoids in the range of sizes from 10 to $100 \mathrm{~s}$ of $\mu \mathrm{m}$ typically found in experiments, estimates for the ratio of some average of the elastic constants and the surface tension as well as the ratio of the surface anchoring strength and the surface tension were obtained. ${ }^{8,13,15,16}$ The latter was consistently found to be significantly larger than predicted by theory and computer simulations. ${ }^{10,17-21}$ This has been attributed to the effects of curvature, but this is by no means certain. ${ }^{22}$

Additional information on the material properties of tactoids can be obtained by means of an externally applied electrical or magnetic field because these couple to their shape and director field..$^{5,23,24}$ Recently, we showed that tactoids formed in suspensions of sterically stabilized "plate-like" gibbsite particles in bromotoluene respond to an increasing magnetic field not by a continuous deformation but by a sudden rearrangement of the director field if the field strength exceeds a critical value, a phenomenon reminiscent of a Frederiks transition. Free floating tactoids of elongated particles do not exhibit a Frederiks transition in a magnetic field: rather they 
reorient and at sufficiently high field strength become slightly stretched along the field direction. ${ }^{5}$

The difference in the behavior of rod- and plate-like colloidal particles is due to different anchoring conditions to the interface between co-existing isotropic and nematic phases. While colloidal rods favor parallel anchoring to the interface, colloidal plates prefer homeotropic anchoring, resulting in spherical tactoids with a radial director field and a hedgehog defect in the center of the droplet. Not surprisingly, the spherical symmetry of these tactoids leads to fundamentally different behavior in an external field. For instance, radial tactoids of particles with a positive magnetic anisotropy should transform to an axial field with ring defect according to theoretical calculations ${ }^{25,26}$ and Monte Carlo simulations. ${ }^{11}$

Interestingly, about ten years ago, Mkaddem and Gartland $^{27}$ investigated defects in radial tactoids within Landau-de Gennes theory and, apart from the familiar radial hedgehog and the (small) ring disclination, reported on the existence of what they termed the split-core defect structure. This configuration, in essence a radial point defect extended to a straight disclination line defect, was found to be metastable. However, the authors suggested that it could be stabilized in a magnetic field, provided the particles have a negative magnetic anisotropy. In this paper, we confirm that tactoids in dispersions of gibbsite platelets, which have a negative magnetic anisotropy, do indeed exhibit this remarkable defect structure at sufficiently high magnetic-field strengths. The associated Frederiks-like transition is caused by a tension characteristic of a disclination line, a tension that has to be overcome by the action of the magnetic field.

Here, we extend our earlier work on tactoids of sterically stabilized gibbsite platelets in an apolar solvent (bromotoluene) to charge-stabilized ones in the polar solvent water. ${ }^{23}$ As we shall see, the sterically and charge-stabilized gibbsite systems represent fundamentally different systems governed by a strong versus a weak surface anchoring of the director field. In the former, the formation of the split-core defect is associated with an elongation of the tactoids, while for the latter this is not so. A macroscopic model accounting for the Frank elastic energy, magnetic energy, and surface and anchoring energies rationalizes our findings and allows us to extract from our measurements the splay-elastic constant and the ratio of the anchoring strength and surface tension.

Analysis of the data from our earlier experiments on tactoids of sterically stabilized gibbsite in terms of the present model produces values for the splay-elastic constant and surface tension that are in agreement with those obtained from a much cruder model. ${ }^{23}$ This reconfirms our earlier conclusion that the isotropic-nematic surface tension obtained from the tactoid shape considerably differs from that found from capillary rise experiments. ${ }^{28}$ We have no explanation for this discrepancy.

\section{MATERIALS AND METHODS}

\section{A. Particle preparation and characterization}

Colloidal gibbsite $\left(\gamma-\mathrm{Al}(\mathrm{OH})_{3}\right)$ platelets were synthesized by hydrothermal treatment of aluminum alkoxides in an acidic environment by the method developed in our laboratory. ${ }^{29}$ For the experiments in apolar solvent, the obtained particles were coated with a modified poly-isobutylene (Shell, SAP 230) (Ref. 30) according to the procedure of Van der Kooij et al., ${ }^{31}$ and were dispersed in 2-bromotoluene. The gibbsite system in water was prepared by coating the bare particles with $\mathrm{Al}_{13}$ polycations, by addition of aluminum chloro hydrate (ACH or Locron P, Hoechst, Germany), to enhance the particle stability by an increase of the surface charge. ${ }^{32}$ Subsequently, the particles were redispersed in a $10^{-2} \mathrm{M}$ sodium chloride solution. Both systems were characterized with transmission electron microscopy (TEM, Tecnai 10), which resulted in a particle diameter of $220 \mathrm{~nm}( \pm 22 \%)$ for the sterically stabilized gibbsite and $207 \mathrm{~nm}( \pm 35 \%)$ for the charged gibbsite platelets.

\section{B. Sample preparation}

Both gibbsite systems were concentrated by centrifugation and subsequent redispersion to attain a concentration in the range where the isotropic and nematic phases coexist, which was $21 \mathrm{v} / \mathrm{v} \%$ for the sterically stabilized gibbsite and $24 \mathrm{v} / \mathrm{v} \%$ for the charged gibbsite. The particle number density in the nematic phase, $\rho$, was estimated to be around $4 \cdot 10^{20} / \mathrm{m}^{3}$ in both systems. Samples of the homogenized dispersions were prepared in flat glass capillaries (VitroCom, $\mathrm{NJ}$ ) with internal dimensions of $200 \mu \mathrm{m} \times 4 \mathrm{~mm} \times 4 \mathrm{~cm}$. The capillaries were filled by capillary action, subsequently flame-sealed, and glued to avoid evaporation of the solvent. In a few hours tactoids appeared and coalesced to form the nematic phase. Measurements were performed near the end of the phase separation process, typically after a few days to weeks, to minimize the effect of flow fields on the tactoid shape and director field.

\section{Magnetic field experiments}

A magnetic field was applied to the samples by means of a Bruker Electromagnet, equipped with large flat pole shoes to ensure a homogeneous field. Field strengths ranged from $B=0-2$ Tesla (T) and were monitored with a LakeShore 421 Gaussmeter. Previous optical and small angle x ray scattering experiments on suspensions of sterically stabilized gibbsite in toluene resulted in a diamagnetic susceptibility anisotropy $\Delta \chi$ of $-10^{-22} \mathrm{~J} / \mathrm{T}^{2}{ }^{33}$ This means that the platelets prefer alignment with the director perpendicular to the magnetic field. The diamagnetic anisotropy has only been measured in systems of sterically stabilized gibbsite, but we expect that the charge-stabilized gibbsite system used here will have the same value, because the platelets are of comparable size.

\section{Polarized light microscopy}

Tactoid shape and director field were directly observed in the magnetic field with a polarized light microscopy setup, assembled from the head of a Zeiss Axiolab microscope, equipped with crossed polarizers, a $10 \times$ and $20 \times$ Nikon CFI 


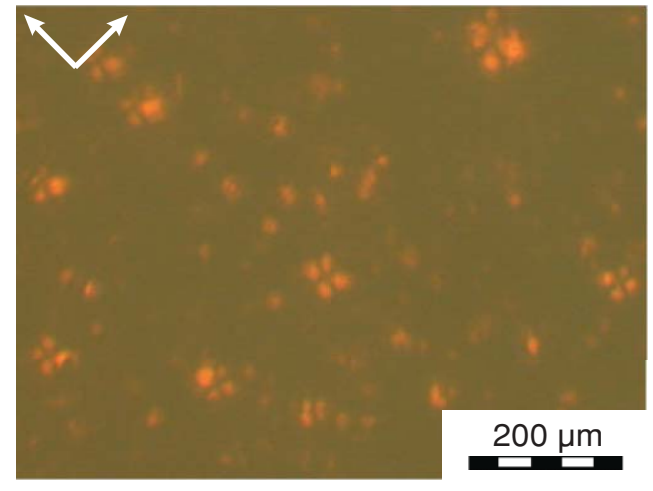

FIG. 1. Polarization light micrograph showing nematic tactoids of various sizes and director-field configurations in an aqueous suspension of charge-stabilized gibbsite platelets. Arrows indicate the orientation of the polarizers.

Plan Fluor ELWD objective, and a Nikon Coolpix 995 CCD camera. The microscope had its focal plane along gravity, with the magnetic field applied in the horizontal direction parallel to the focal plane. A full wave retardation plate $(\lambda$ $=530 \mathrm{~nm}$ ) was used to determine the orientation of the director. The nematic phase of gibbsite platelets in water has a negative birefringence, which means that the largest refractive index is perpendicular to the director. ${ }^{34}$ As a consequence, insertion of the full wave retardation plate will lead to a color shift to orange if the director is parallel to the slow axis of the plate and to blue in the case of a perpendicular orientation.

\section{EXPERIMENTAL RESULTS}

A typical polarized light micrograph of tactoids in an aqueous suspension of our gibbsite platelets is given in Fig. 1, showing a group of tactoids of different size and director-field configuration. From groups like the one shown in Fig. 1, we investigated the response of representative droplets of different size to the application of a magnetic field.

Figure 2 shows how two large tactoids with a radius of $\approx 19$ and $30 \mu \mathrm{m}$ are perturbed by an externally applied magnetic field. At zero field, the tactoids are spherical and the extinction pattern corresponds to that of a 3D radial director field emanating from a hedgehog point defect in the center of the tactoid, ${ }^{23,35}$ also see the schematic representation of Fig. 7(e). This follows from the observation that the diagonal extinction cross, associated with the region where the platelets are aligned parallel to the polarizers, co-rotates with the polarizers.

However, if the magnetic field strength is larger than some critical value that according to our observations seems to depend on the radius of the tactoid, the dark diagonal cross transforms into a horizontal band. The fact that the dark band is aligned along the magnetic field direction, itself inclined at $45^{\circ}$ relative to the polarizers, implies that in that region we must be viewing the gibbsite platelets face on. In this configuration, the particles do not exhibit any birefringence. Such a configuration makes sense, of course, once we realize that the negative magnetic diamagnetic susceptibility tends to align the platelets perpendicular to the magnetic field direction. With this in mind, we can now interpret the transformation of extinction patterns in terms of a transformation of the point defect into a disclination line, ending in what must be the two halves of a point defect, in the direction of the magnetic field.

This implies that from this line defect the director-field lines must run radially to the droplet's interface, resulting in a $2 \mathrm{D}$ radial director field in the middle part of the droplet, while the director field on both sides of this structure remains radial in 3D. A schematic of the envisaged director-field structure is given in Fig. 7(f). So, the emergence of the dark horizontal band in the direction of the magnetic field must be due to the alignment of the platelets perpendicular to the magnetic field: directly looking at the core of the disclination line the director field points into the focal plane and in this orientation the particles do not display any birefringence.
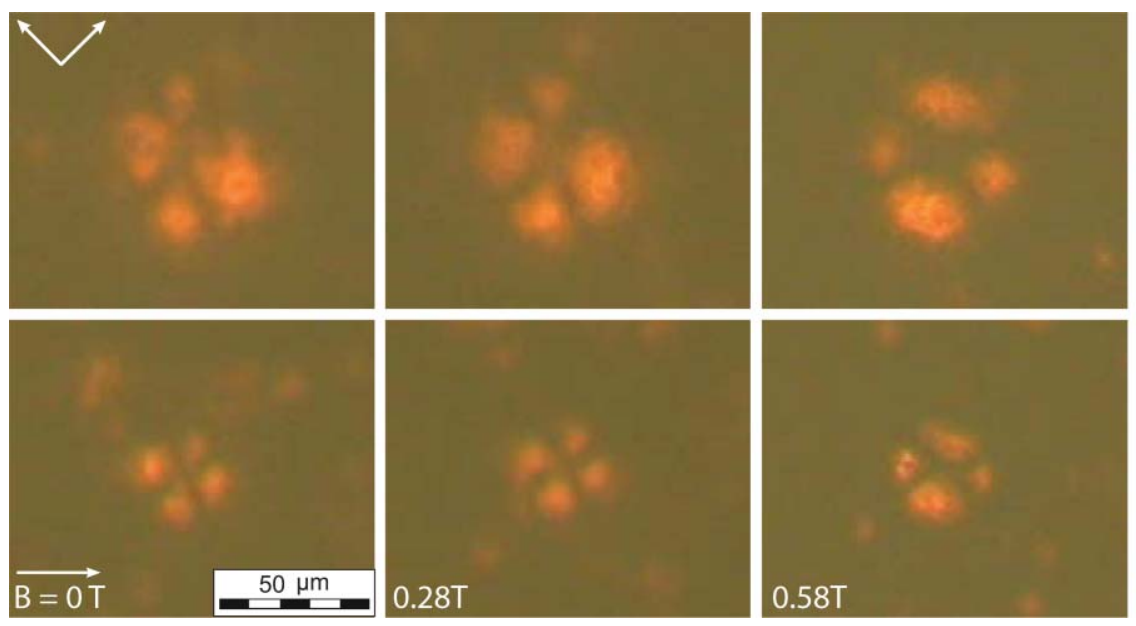
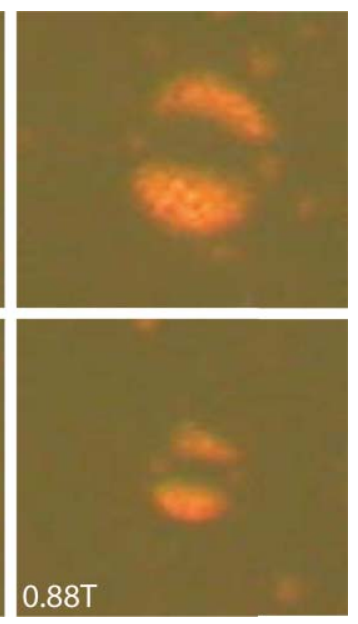

FIG. 2. Polarization microscopic images, showing tactoids of approximately $30 \mu \mathrm{m}$ (top row) and $19 \mu \mathrm{m}$ (bottom row) radius in a dispersion of chargestabilized gibbsite platelets in water for a series of increasing magnetic field strengths indicated. The orientations of the polarizers and the magnetic field are indicated by arrows in the upper-left corner, and the magnetic field with the horizontal arrow in the lower-left corner. The extinction pattern changes fairly abruptly with increasing field strength. As discussed in the main text, we attribute this to the transformation of a radial director field characterized by a point defect at low magnetic field strength to a so-called split-core line defect that increases in length with increasing magnetic field strength. 


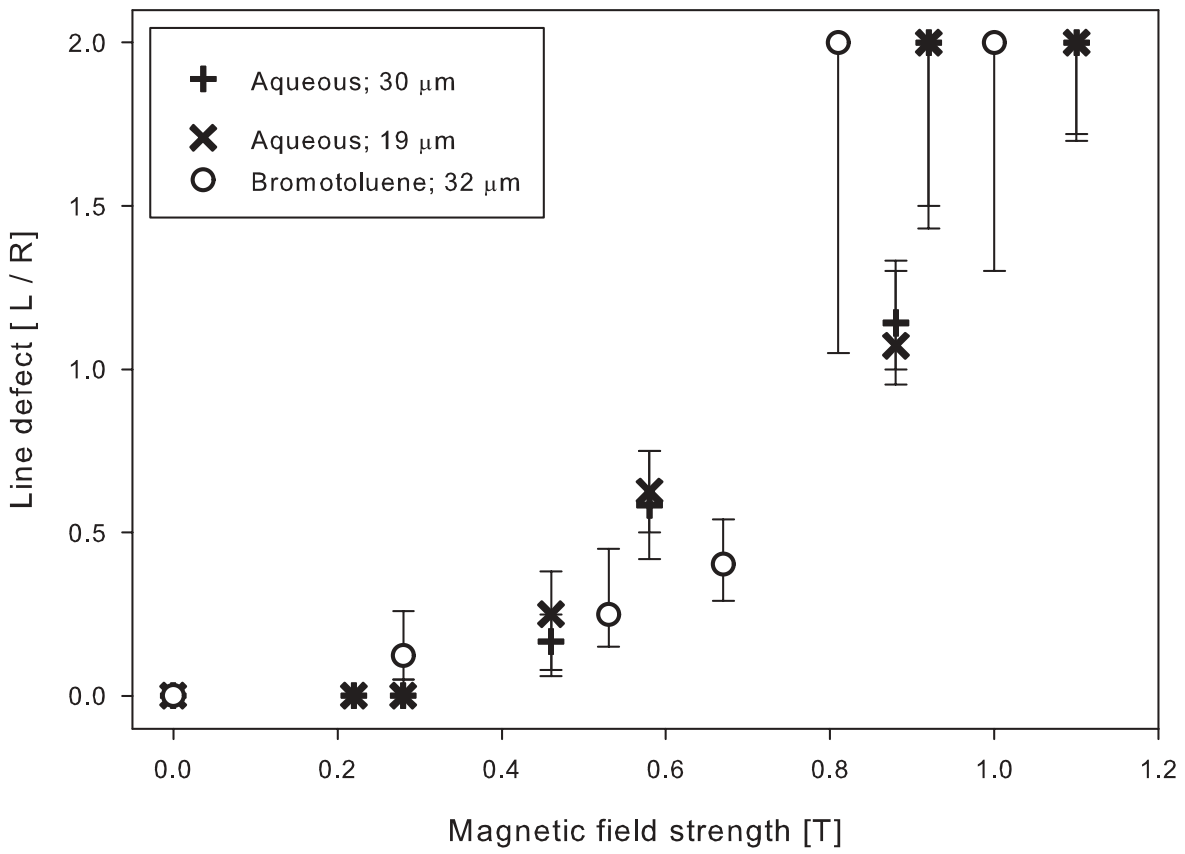

FIG. 3. Length of the line defect as a function of magnetic field strength, for tactoids of charge-stabilized gibbsite in water (crosses and pluses) and sterically stabilized gibbsite in bromotoluene (circles). Indicated in the box are the (initial) radii of the tactoids. The length of the split-core defect, deduced from the length of the extinction band along the magnetic field shown in Fig. 2, is scaled in the aqueous system to the tactoid radius and in bromotoluene with half the tactoid length in the direction of the line defect. See the main text for an explanation of how the line-defect length was exactly measured. We note here that tactoids in bromotoluene do not remain spherical but elongate in the field direction.

It is of some interest to point out that this director-field structure corresponds to that associated with the so-called split-core defect, the existence of which in nematic drops was predicted ten years ago by Mkaddem and Gartland albeit only as a metastable state. ${ }^{27}$ These authors also suggested, however, that the split-core defect could be stabilized by the application of a magnetic field but only if the nematogens have a negative diamagnetic susceptibility. This exactly corresponds to the situation that we have investigated. So, our measurements confirm that the spit-core defect structure, which as far as we are aware has never previously been observed, can indeed be made stable in a magnetic field, in agreement with theoretical prediction.

Upon a further increase of the magnetic field, the length of the line defect increases until its length spans the entire tactoid width. For the two tactoids of different radii of Fig. 2, this happens at a field strength of approximately $1 \mathrm{~T}$. If we now rotate the polarizers to a vertical-horizontal orientation (not shown), the horizontal band remains dark while the birefringence of the regions above and below this band completely disappears, indeed corresponding to a $2 \mathrm{D}$ radial director field configuration in the entire droplet.

The length of the line defect relative to the radius of the tactoid is shown in Fig. 3, as a function of the magnetic field strength. In contrast to thermotropic liquid crystals where line defects can actually be observed, in this system the line-defect length has to be deduced from the extinction pattern, which is not trivial. We have determined the length of line defect by extending the diagonal extinction bands shown in Fig. 2 until they cross the horizontal extinction band along the magnetic field and then measured the distance between these crossing points. The indicated error bars were determined from the distance of these points to the ends of the horizontal dark band. Although the size of these tactoids differs by a factor of 1.6, the transition from a radial to a split-core type director field seems to take place in the same admittedly somewhat broad range of about 0.3 to $0.6 \mathrm{~T}$. On the other hand, the director-field structures observed at $1.1 \mathrm{~T}$, where for both drops the defect lines seem to traverse the entire drop, are slightly different. The dark horizontal band of the largest tactoid of the two seems slightly wider in the middle, while it is much more uniform in the smaller tactoid. This might indicate that the director field of the largest tactoid is not a purely 2D splay field but may exhibit a slight bend deformation as well.

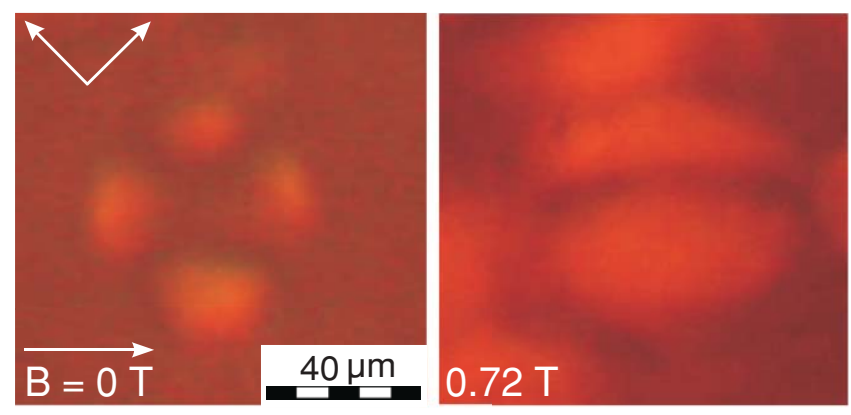

FIG. 4. Polarization micrographs of a nematic tactoid of approximately $31 \mu \mathrm{m}$ radius of sterically stabilized gibbsite platelets suspended in the apolar solvent bromotoluene. Indicated are the orientations of the polarizers (arrows at right angles in the top left-hand corner) and the magnetic field direction (lower left-hand corner). Left: at zero magnetic field the extinction pattern is consistent with a radial director field and a point defect in the center. Right: an applied magnetic field of $0.72 \mathrm{~T}$ transforms the point defect into a line defect and the tactoid becomes elongated in the field direction. 


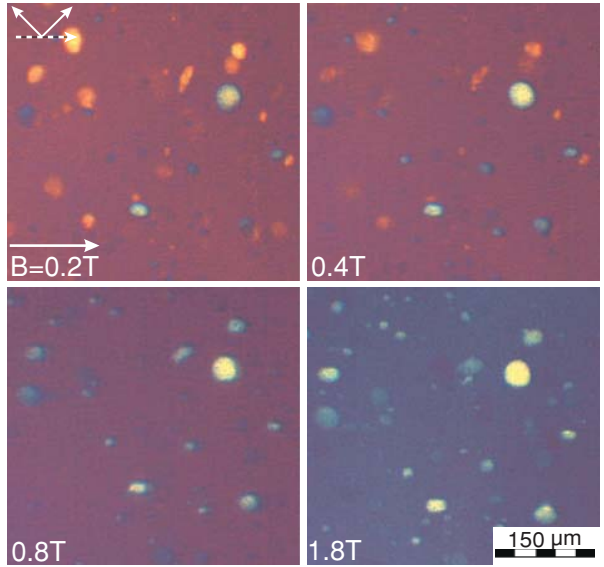

FIG. 5. Polarization microscopic images showing tactoids of chargestabilized gibbsite in water with a uniform director field in a magnetic field. Indicated are the orientations of the polarizers (arrows at right angles in the top left-hand corner), retardation plate (dashed arrow), and the magnetic field direction (horizontal arrow). Different colors represent different director orientations, as explained in the main text. The isotropic background aligns in the magnetic field and the tactoids rotate with increasing field strength to orient their director perpendicular to the field direction.

Previous experiments have shown that tactoids of sterically stabilized gibbsite in the apolar solvent bromotoluene display a similar transformation with increasing magnetic field observed in the aqueous system ${ }^{23}$ (see Fig. 4). For comparison, we have plotted in Fig. 3 the length of the splitcore defect in tactoids of sterically stabilized gibbsite as a function of magnetic field strength, in this case scaled with half the length of the droplet in the direction of the line defect.

It is important to stress that there is a significant difference between the behavior of tactoids in the two types of solvent. For the sterically stabilized gibbsite platelets in bromotoluene, the formation of the split-core defect is accompanied by an elongation of the droplet in the field direction [see Fig. 7(g) for a schematic representation]. This elongation starts at a slightly higher magnetic field strength than the "stretching" of the point defect into the line defect that we believe to be the split-core defect. In Ref. 23, the tactoid elongation was explained by presuming a rigid anchoring of the director field to the interface of the tactoids. The fact that tactoids of the present system of charge-stabilized gibbsite in water remain spherical, despite the emergence of a growing split-core defect with increasing field strength, implies that in this case the anchoring of the director field to the interface must be much weaker. We return to this important issue in our discussion below.

Interestingly, we find that very small tactoids behave rather differently from the larger ones, and in fact do not exhibit the typical Maltese cross extinction pattern typical of a radial director field. This is shown in Fig. 5, presenting a micrograph series of tactoids up to approximately $40 \mu \mathrm{m}$ in diameter in a magnetic field of increasing strength. A retardation plate was used here indicated by the dashed arrow, pointing along the magnetic field direction. At zero field (not shown), these tactoids have different but otherwise uniform colors (orange and blue tints). This means that the tactoids have a uniform director field and different orientations with
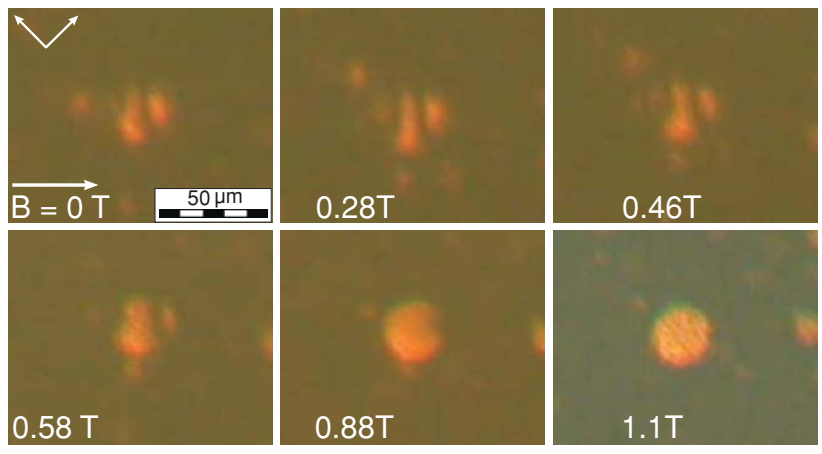

FIG. 6. Polarization micrograph of an intermediate-sized tactoid with increasing magnetic field strength. Arrows in the upper-left corner indicate the orientation of the polarizers, and the magnetic field direction is indicated by the horizontal arrow. At zero field, the extinction pattern is consistent with an axial director field and a ring defect close to the top of the droplet, where we note that gravitational force points downward along the vertical [see Fig. 7(c)]. The central part of the tactoid, where the director is more or less uniform and oriented along the vertical direction, becomes larger with increasing field strength until the entire tactoid has a uniform director field.

respect to the retardation plate. For orange ones, the nematic director is oriented parallel and for the blue ones, perpendicular to the retardation plate. With increasing magnetic field strength several changes can be observed.

First, the color of the isotropic background shifts from the magenta-red of the retardation plate to a blue tint. This indicates that the isotropic background phase aligns in the magnetic field and forms a para-nematic phase with the director perpendicular to the retardation plate and the field direction. Second, the color of some of the tactoids turns from orange into blue, indicating that the nematic director rotates from an orientation parallel to the field to that perpendicular to it. Some of the orange-colored tactoids seem to (almost) disappear from view, which is plausibly due to their alignment perpendicular to the focal plane in which case they do not exhibit any birefringence. Blue tactoids display a color shift toward green/yellow, plausibly caused by a slight rotation of the director to a more vertical orientation and possibly also to an increase of the nematic order parameter. Note that tactoids also appear blue if the orientation is not completely perpendicular to the retardation plate. Finally, some of the tactoids seem to become slightly elongated in the field direction.

As we shall show in Sec. IV, these observations can be rationalized from the standard continuum theory of nematics. Before we turn to that, we end this section with a brief discussion of how different director fields transform themselves from one to the other.

To this end, we present in Fig. 6 a tactoid with a directorfield structure in between the radial and uniform director fields typical of large and small tactoids, respectively. The transition from a radial tactoid to that with a uniform director field, we imagine, can proceed via two different routes. Plausibly, this may happen either via the continuous displacement of the point defect from the center of the drop to a position outside it where it becomes virtual and eventually move to infinity, or via a transition of the point defect to a ring defect with increasing radius that also becomes virtual if it leaves the 


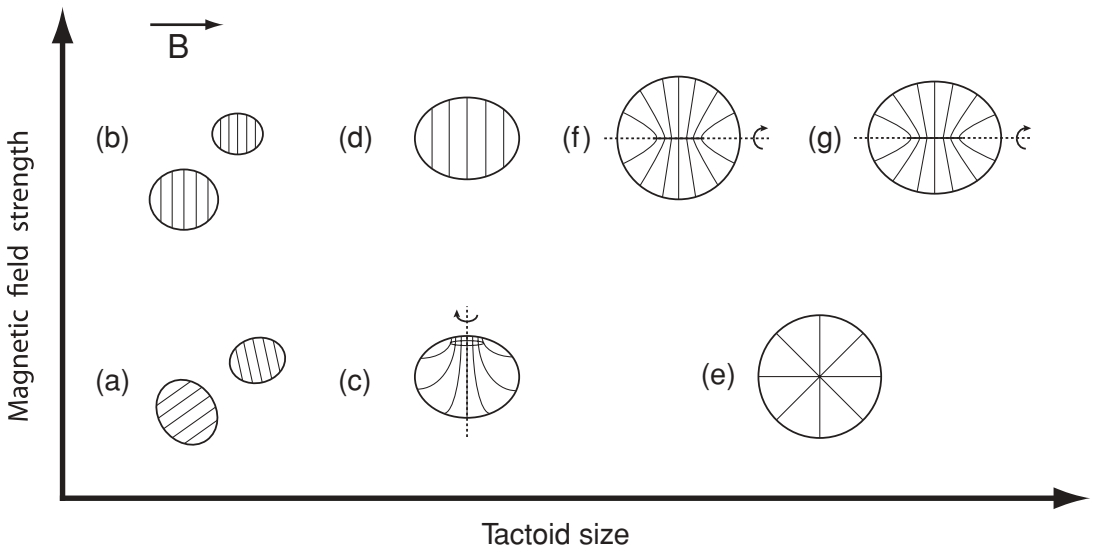

FIG. 7. Schematic overview of tactoid shapes and director-field configurations observed in dispersions of gibbsite in water (a-f) and in bromotoluene (e-g), as a function of the tactoid size and the magnetic field strength. The arrow indicates the direction of the magnetic field. Note that the main body axis of the platelets (the surface normal) points in the mean along the director (see Fig. 8).

droplet. This shall be discussed more extensively in a forthcoming paper. $^{35}$

The director field of the particular tactoid studied here is schematically depicted in Fig. 7(c) and represents the route where the point defect opens up to become a ring defect with a radius that increases as the drop size decreases. Note that the tactoid sediments toward the nematic phase and that presumably due to the flow fields this ring defect we always find to be on the rear of the drop with respect to the direction of translation. ${ }^{36}$ If a magnetic field is applied to this tactoid, the size of the birefringent part in the center of the tactoid increases with increasing magnetic field strength, which implies that the radius of the ring defect must increase accordingly. This continues until the director field becomes uniformly oriented at a right angle to the magnetic field at a magnetic field strength of 1.1 T. So, the transformation from a nonuniform to a uniform director field with increasing magnetic field strength seems to proceed in a similar way to that in the absence of an external field with decreasing tactoid size.

To summarize our findings, we refer to the overview of the observed director-field structures in Fig. 7. Different transitions or crossovers can be distinguished as a function of tactoid size, magnetic-field strength and solvent type. With increasing magnetic field we find for: (1) small tactoids with uniform director field (a) a reorientation to have their directors perpendicularly positioned to the magnetic field (b); (2) intermediate sized tactoids with a ring defect (c) a transformation to a uniform director field (d); (3) large radial tactoids with a hedgehog defect (e) a transformation to a split-core defect with the drops remaining spherical in a polar solvent (f), or adopting an ellipsoidal shape in an apolar solvent $(\mathrm{g})$.

In Sec. IV we put forward an approximate theory that explains our observations. As we shall see, the theory in fact enables us to extract from the experiments useful physical parameters of the system in hand.

\section{THEORY}

It appears that the director field of nematic tactoids in dispersions of gibbsite particles is not fixed but responds to both their size and the strength of the magnetic field. In addition, tactoids of sterically stabilized gibbsite in bromotoluene become elongated in a sufficiently strong magnetic field, while those in dispersions of charge-stabilized gibbsite in water remain spherical. In order to rationalize these observations, summarized in Fig. 7, we present a macroscopic theory based on a competition between the elastic, surface, and magnetic properties of drops of a nematic phase freely floating in an isotropic phase.

Our free energy $F=F_{E}+F_{S}+F_{M}$ of a nematic drop of given volume $V$ consists of three contributions:

(1) The Oseen-Frank free energy of a deformed director field $\vec{n}(\vec{r})$ that in the one-constant approximation reads

$$
F_{E}=\frac{1}{2} K \int d^{3} \vec{r}\left[(\vec{\nabla} \cdot \vec{n})^{2}+(\vec{\nabla} \times \vec{n})^{2}\right],
$$

where $K$ is some average of the elastic constants. In our calculations we shall presume only pure splay deformations, in which case $K=K_{1}$, i.e., the splay-elastic constant. The integration over the spatial co-ordinate $\vec{r}$ is implied to be taken over the entire volume $V$ of the drop.

(2) For the interfacial energy we presume a form of the Rapini-Papoular type, ${ }^{37}$

$$
F_{S}=\int d^{2} \vec{r}\left[\gamma+w-w(\vec{q} \cdot \vec{n})^{2}\right],
$$

where the integration is now implied over the entire surface of the drop. Here, $\gamma$ is the bare surface tension of the drop, $w$ an anchoring free energy per unit area, $\vec{q}=\vec{q}(\vec{r})$ the surface normal, and $\vec{n}$ the director field at the interface. Platelets for entropic reasons prefer homeotropic alignment, ${ }^{28}$ so $w>0$.

(3) The magnetic energy of a nematic drop obeys ${ }^{38}$

$$
F_{M}=-\frac{1}{2} \rho \Delta \chi \int d^{3} \vec{r}(\vec{n} \cdot \vec{B})^{2},
$$

where $\rho$ is the particle number density, $\Delta \chi$ the diamagnetic susceptibility anisotropy, and $\vec{B}$ the magnetic field vector. Our gibbsite platelets have a negative magnetic susceptibility, so $\Delta \chi<0.33,39$ 


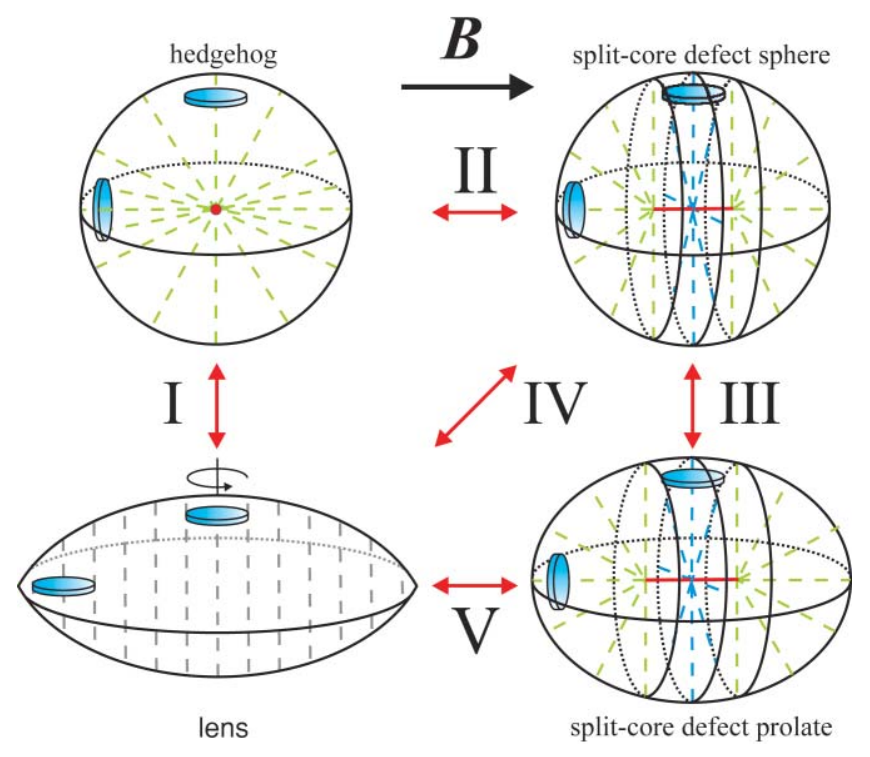

FIG. 8. Schematic representation of the main droplet shapes and director fields, indicated by dashed lines, consistent with our observations. The competition between homeotropic surface anchoring, surface tension, elastic deformation, and the magnetic field $B$ gives rise to transitions between at least four types of tactoid, indicated by the Roman numerals I-V. The radial director field of the spherical tactoid with a hedgehog defect (top left) has perfect anchoring and minimal surface tension but has a suboptimal magnetic energy. Replacing the point defect by split-core line defect (top right) lowers the magnetic energy but increases the anchoring energy. Elongating the splitcore droplet lowers the magnetic and anchoring energy at the expense of the surface energy. The lowest magnetic energy has a tactoid with a uniform field (bottom left). Its shape minimizes the sum of the anchoring and surface energies, for homeotropic anchoring producing a lens-shaped object. The disks are not drawn to scale and serve to indicate the platelet orientations in the various drops.

In principle, we would need to minimize the free energy with respect to the director field and shape of the interface, given the fixed volume of the drop and given that the norm of the director is by definition equal to unity. This produces a constrained free boundary value problem that is very hard to solve, even numerically. So, we choose to simplify the problem by presuming the director field either to be uniform or to consist of a pure splay type. For a uniform field we apply the well-known Wulff construction ${ }^{40}$ to find the optimal shape. For the pure splay field we presume a line defect of given length to be in the center of the drop that in turn we presume to be of prolate shape, i.e., to be an ellipsoid of revolution. The director field over the entire length of the disclination line is presumed to be a $2 \mathrm{D}$ radial splay field, while in the remainder of the drop we presume a 3D splay field emanating from the split-core defect at the ends of the disclination line. We optimize both the length of the line defect and the aspect ratio of the drop. We perturbatively do this for aspect ratios near unity, i.e., for drops that are not very elongated, in line with our observations. Stability diagrams are calculated by comparing free energies of various types of drop (schematically presented in Fig. 8).

In the following, only an outline of the main results of our model are presented. A detailed account of our calculations, which are quite nontrivial, shall be given elsewhere. ${ }^{41}$ We summarize our results in stability diagrams in Fig. 10 that show the optimal tactoid shape and director field as a function of the three for the problem relevant dimensionless groups associated with the tactoid size, the anchoring strength of the director field the tactoid's surface, and the magnetic-field strength. These dimensionless groups are $\Re \equiv R \gamma / K_{1}$, where $R$ is the radius of the equivalent spherical drop, $\omega \equiv w / \gamma$ a dimensionless anchoring strength, and $\beta^{2} \equiv-\rho \Delta \chi B^{2} K_{1} / \gamma^{2}$, with $B$ the strength of the magnetic field. There is also a fourth dimensionless group, $\aleph \equiv b \gamma / K_{1}$, associated with the core of the line defect the radius of which is presumed to be of order $b$. As $b$ must be a microscopic length in lyotropic nematics, ${ }^{42,43}$ i.e., on the scale of the diameter of the platelets, its magnitude can only be estimated. ${ }^{23}$ Fortunately, $\aleph$ enters our equations only logarithmically so our results depend only very weakly on its value.

Before discussing our phase diagrams in more detail, it is useful to explicitly consider the driving forces for the transitions between the different tactoid shapes and director-field configurations presented in Sec. III and schematically shown in Fig. 8. We can distinguish between droplets with perfect and imperfect planar alignment of the platelets to the surface, corresponding to perfect and imperfect homeotropic anchoring (see Fig. 8). Clearly, a drop with a uniform director field has an imperfect anchoring. In this case, there is no deformation of the director field but also the magnetic energy is minimal because the drop is able to orient and align itself in the magnetic field. The alignment of the director field is perpendicular to the magnetic field on account of the negative diamagnetic susceptibility of the particles.

We conclude that for a drop with a uniform field the only free-energy cost is that associated with the imperfect surface anchoring, which incidentally can be minimized further by deforming the shape of the drop. Because of the preferred homeotropic surface anchoring the tactoids tend to become oblate shaped and flatter the larger dimensionless anchoring strength $\omega \equiv w / \gamma>0$, the sole parameter that dictates the shape of a drop with a uniform director field. Invoking the Wulff construction, ${ }^{40}$ we find that for $\omega \geq 1$ the oblate in fact transforms to a lens with a sharp rim. Interestingly, the difference in free energy between the exact Wulff shape and a lens constructed from two cut spheres and optimized with respect to the cutting angle is minute (less than $0.5 \%$ ) for all values of $\omega$, so for simplicity we used this lens shape for constructing our stability diagrams.

For spherical tactoids, perfect or "strong" anchoring conditions are fulfilled by a three-dimensional radial director field that radiates outward from a hedgehog point defect in the center of the drop (see Fig. 8). For this configuration, anchoring is ideal but the director field is distorted and the orientation of a part of the particles is suboptimal relative to the magnetic field that, again, prefers to align them perpendicular to it. The hedgehog director-field configuration wins out over the uniform one if the product $\Re \times \omega=R w / K_{1}$ is large enough by an amount that is set by the dimensionless group $\beta^{2} \equiv-\rho \Delta \chi B^{2} K_{1} / \gamma^{2}$. In zero magnetic field this happens if $\Re \omega>3$ for spherical drops and a slightly higher value for the transition from the lens to a spherical hedgehog (see Fig. 9). This implies that if we observe the transition for a given tactoid size and if we establish the value of the elastic constant 


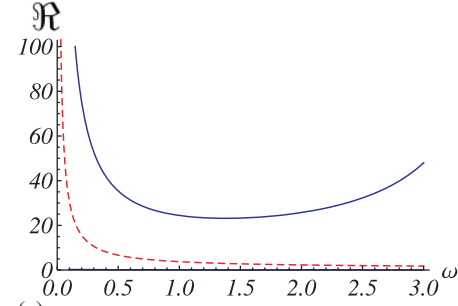

(a)

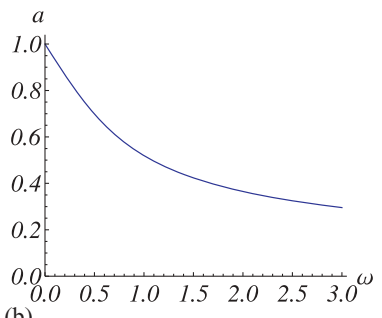

(b)

FIG. 9. (a) The critical value of the dimensionless tactoid size $\mathfrak{R} \equiv R \gamma / K_{1}$ for the transition from a uniform to a radial director field as a function of the dimensionless anchoring strength $\omega \equiv w / \gamma$. Indicated by the dashed line is the critical value at zero magnetic field with $\beta^{2} \equiv-\rho \Delta \chi B^{2} K_{1} / \gamma^{2}=0$, and the solid line shows the limit for a very strong magnetic field where the defect is stretched through the entire tactoid $(L / R=2)$, which is the vertical asymptote (dotted line) in the phase diagram in Fig. 11. (b) The aspect ratio $a$ of the lens shape, i.e., the ratio of its thickness to its diameter, is completely determined by $\omega$, and for the values in the phase diagram, i.e., $\omega \in(0.25,2)$, it ranges from 0.84 to 0.36 .

independently, this inequality produces a lower bound for $w$. We return to this in Sec. VI.

It follows that the hedgehog configuration is not favorable for a sufficiently small tactoid. ${ }^{2,3}$ For a strong enough magnetic field it cannot be maintained either, because then the magnetic energy penalty becomes prohibitively large. To allow more particles to comply with the preference imposed by the magnetic field, the drop can either adopt a uniform director field at the expense of imperfect anchoring or stretch the point defect to a line defect of topological charge +1 in the direction of the magnetic field (see Fig. 8). In this split-core defect structure discussed in the preceding sections, perfect surface anchoring cannot be maintained albeit that it can be reduced by elongating the drop. This can obviously only happen at the expense of a surface energy. In comparison with the hedgehog configuration, the split-core configuration has a lower magnetic energy penalty, which may even become zero if the defect line runs through the entire tactoid but it does have a higher elastic deformation energy, a nonzero anchoring penalty, and potentially a higher surface energy.

In our numerical calculations summarized in Fig. 10, we set the value of the dimensionless anchoring strength equal to $\omega=0.25,0.5,1$, and 2 , where we note that a value around $\omega=0.5$ was deduced from capillary-rise experiments for sterically stabilized gibbsite platelets in toluene. ${ }^{28}$ In the limit of $\omega \rightarrow 0$ the free-energy cost of increasing of the surface area is infinite, implying that the tactoids remain spherical. The stability diagrams presented in Fig. 10 show that, as expected, a sufficiently small tactoid has a homogeneous director field for any magnetic field strength because of the predominance of the Frank elastic energy. Larger tactoids adopt a hedgehog configuration for weak magnetic fields and a line defect that runs through the entire drop for very large magnetic fields, on account of the predominance of the anchoring energy. As advertised, the cross-over size in the absence and presence of a strong magnetic field can provide information on the anchoring strength and its dependence on $\omega$ is shown in Fig. 9.

How the transition between a uniform and a hedgehog configuration proceeds we do not know for certain but it may involve either a hedgehog defect or a small ring defect that moves in from outside of the drop, where it is virtual. Indeed, in Sec. III we have seen configurations with a defect that is close to the tactoid's surface. However, if we model a 3D radial director field emanating from a point defect that is off center, then it turns out that this configuration is always energetically more expensive than the central hedgehog or the lens with a uniform field. ${ }^{35}$ Therefore, we conclude that the tactoid with boundary defect must be a metastable configuration and because of its relatively high free energy is absent in our phase diagram. We speculate that the observed configuration is stabilized by the flow field caused by the sedimentation. ${ }^{36}$

The second transition we find in the diagrams is that of spherical drops with a central hedgehog to those with a split core. This transition happens for a sufficiently strong magnetic field and allows more particles to adopt the magnetically preferred orientation. The critical magnetic field strength $B_{c}$ associated with this Frederiks-type of transition obeys

$$
B_{c}^{2}=\frac{4 K_{1}}{\rho \Delta \chi R^{2}}\left(\log \left(\frac{b}{R}\right)+2\right),
$$

where $b$ is the diameter of the core of the line defect that we expect to be of the order of the plate diameter. This expression differs by a factor of two thirds from our earlier, more approximate theory based on the presumption of perfect anchoring requiring the drop to stretch with increasing length of the split-core defect. ${ }^{23}$ Equation (4) is independent of $\omega$ because at the onset of the transition from hedgehog to split-core defect, the anchoring is still perfectly homeotropic. Given that we now allow for imperfect anchoring, the length of the defect increases with the magnetic field strength without stretching the drop. For very strong fields the line defect stretches throughout the entire tactoid to eliminate the magnetic energy cost altogether.

We find that for all four values of $\omega$ of Fig. 10, there is a region of sizes and magnetic field strengths where tactoids are not spherical or lens shaped, but elongated. These elongated tactoids have a split-core defect along their main axis, and their region of stability is larger for larger values of $\omega$. The reason for this is that the ellipsoid has a lower anchoring energy than the equivalent sphere with line defect. For increasing magnetic field strength a sufficiently large spherical drop with a split-core defect stretches to an ellipsoid. This transition takes place at a magnetic field strength that we denote by $B_{*}$ and is a very complicated expression that we present in a separate publication. ${ }^{41}$ At this critical point the length of the defect line is about half the radius of the drop, $L \approx 0.5 R$. The length of the line defect increases with increasing magnetic field strength (see Fig. 11). The length of the line defect in the elongated drop is larger than that in equivalent spherical tactoid, as follows from the slight downward deflection of the lines of constant $L / R$ in the ellipsoid region.

Interestingly, the aspect ratio of the drops at some point decreases again with increasing magnetic field strength, i.e., depends nonmonotonically on the magnetic field strength. For high enough fields the drops become spherical again (see Fig. 11). This is caused by the change in the line-defect length that affects the balance between the magnetic and anchoring energy. For large enough anchoring energies, elongated 

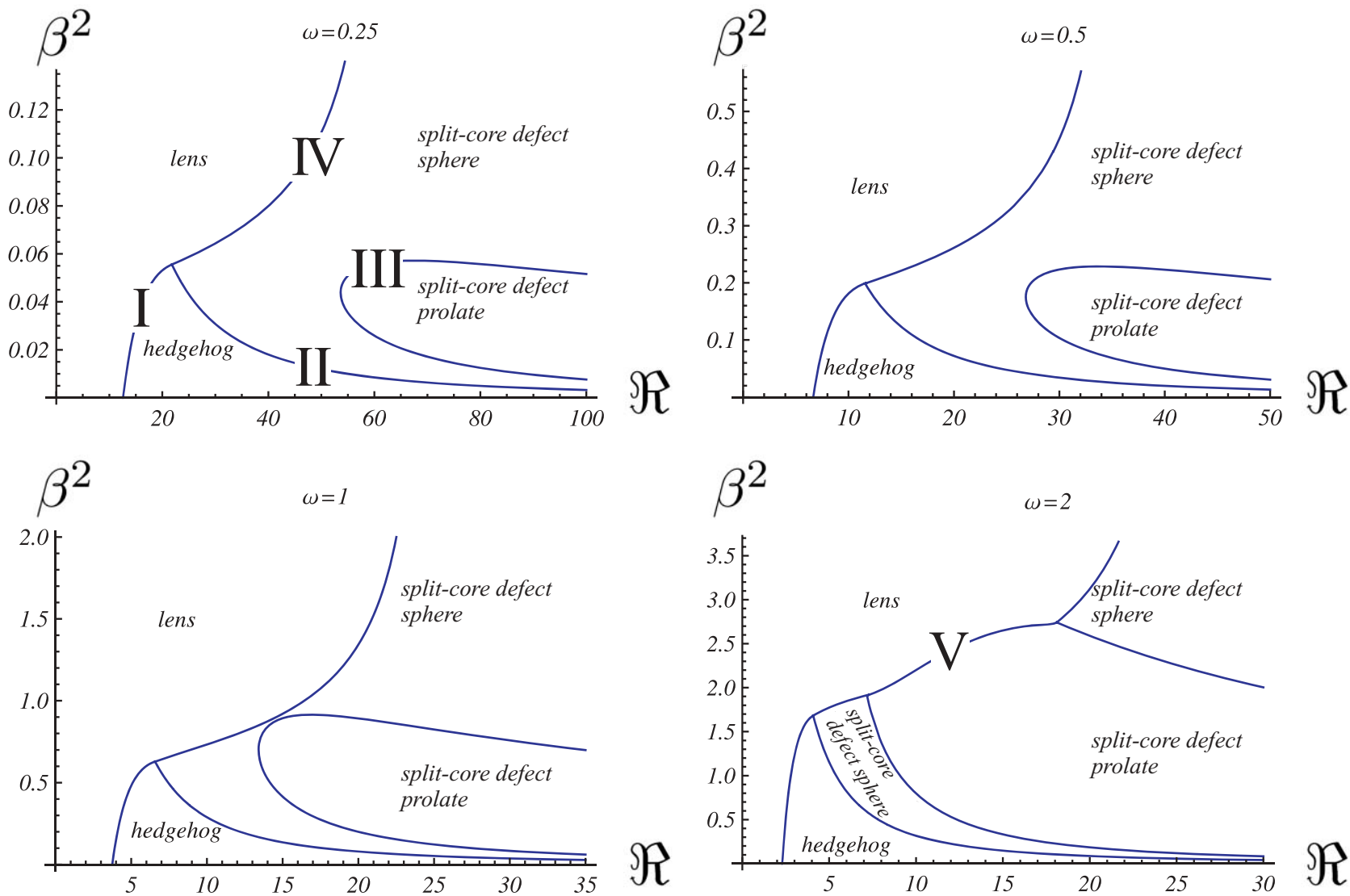

FIG. 10. Phase diagram with a lens-shaped tactoid with uniform field, a spherical hedgehog, a spherical and prolate tactoid, both with split-core defect, for $\omega=0.25$ (top left), $\omega=0.5$ (top right), $\omega=1$ (bottom left), and $\omega=2$ (bottom right). The axes indicate dimensionless measures $\beta^{2} \equiv-\rho \Delta \chi B^{2} K_{1} / \gamma^{2}$ of the magnetic field strength $B$ and $\Re \equiv R \gamma / K_{1}$ of the size of the tactoids (see the main text). The radius $R$ of the spherical drops defines the volume of the tactoids, which for a given $R$ is equal for all configurations. The Roman numerals correspond to the transitions as indicated in Fig. 8. The transitions I, II, III, and IV occur in every diagram, and transition V only occurs for $\omega=2$.

tactoid structures push out spherical hedgehog and split-core tactoids from the phase diagram. The maximum value of the aspect ratio observed is dictated by $\omega$, allowing us to estimate this quantity from experiments.

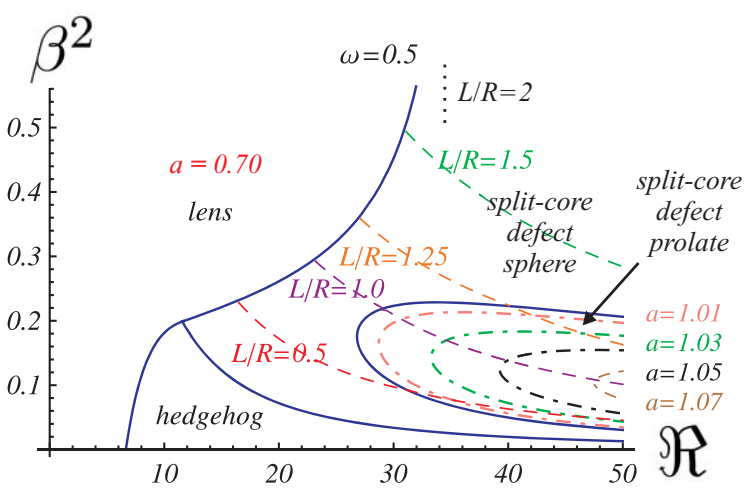

FIG. 11. The phase diagram from Fig. 10 for $\omega=0.5$ with the structural details of the line-defect length and the aspect ratio. The dashed lines indicate a constant defect length $L$, relative to the sphere radius $R$. The dotted line shows the limit of $L / R=2$, i.e., a split-core defect through the entire tactoid. The dashed-dotted lines in the prolate ellipsoid region have a constant aspect ratio $a$, where $a=1$ at the transition from sphere to ellipsoid. The exact shape of the lens depends only on $\omega$ and its aspect ratio is 0.70 for $\omega=0.5$.
In Secs. V and VI we apply the theory to our experimental data on charge-stabilized gibbsite in water. We also reevaluate our previous findings on sterically stabilized gibbsite in bromotoluene. These two cases correspond to very different values of $\omega$ and are examples of weak and strong anchoring.

\section{FITTING PROCEDURE}

If we compare the experimentally observed shape and director-field transitions with those of the theory developed in Sec. IV, we find that all of those shown in Fig. 7 are found in the predicted phase diagrams, except for the transitional structures associated with the point or ring defect near the surface of the tactoid. We believe on theoretical grounds that this configuration is not stable in a quiescent tactoid and may be caused by the flow field resulting from the sedimentation process, because those that we find have them always located on the far side of the moving drop. It is well established that flow fields influence director-field structures in nematic drops. ${ }^{36}$

In fact, we are able to directly apply the theoretical model described in Sec. IV to the experimental data, and extract material parameters relating to our liquid crystal droplets. Of particular use here are the degree of tactoid elongation and length of the split-core defect, obtained as a function of the magnetic field strength and the size of the drops. Because of the 
numerous ways in which the parameters can be determined, we first give an outline of the approach that we have taken, and present the resulting curve fits and parameter values in Sec. VI. Readers not interested in the technicalities of our fitting procedures may wish to move on to Sec. VI.

The two types of systems investigated, i.e., the sterically stabilized gibbsite in the apolar solvent bromotoluene and the charged-stabilized gibbsite in the polar solvent water, turn out to provide us with examples of two limiting cases in terms of the dimensionless anchoring strength $\omega \equiv w / \gamma$. The drops found in the aqueous gibbsite dispersions exhibit no discernable deviation from the spherical shape, implying a high surface tension relative to the anchoring energy, so $\omega \rightarrow 0$. So, in this limit the model is independent of the surface tension $\gamma$ and this necessitates a slight modification of the state diagrams such as plotted in Fig. 10, where on both the horizontal and vertical axes we replace $\gamma$ by $w$, so with the dimensionless groups $-\rho \Delta \chi B^{2} K_{1} / w^{2} \equiv \beta^{2} / \omega^{2}$ and $R w / K_{1}=\Re \omega$ on the axes. For the drops found in dispersions of sterically stabilized gibbsite, the energetic penalty for an increase in the surface area is not that large because they do become visibly elongated at high field strengths albeit not hugely so, implying $\omega \approx 1$.

There are a number of ways to determine the value of the parameters $K_{1}, \gamma$, and $w$ from fitting the model to the experimental data. The data of both the length of the splitcore defect and the tactoid elongation are a function of the magnetic field strength, which means that for the curve fits we have to vertically traverse the phase diagrams for a chosen scaled drop size $\Re \equiv R \gamma / K_{1}$ (for the sterically stabilized system) or $\Re \omega$ (for the charge-stabilized system) that has the best match with the data points of the line-defect length or tactoid aspect ratio. The quality of this match is then determined by choosing the value of the dimension-bearing quantities $B^{2} / \beta^{2}=\gamma^{2} /\left(-\rho \Delta \chi K_{1}\right)$ or $\omega^{2} B^{2} / \beta^{2}=w^{2} /\left(-\rho \Delta \chi K_{1}\right)$ depending on the type of system; and the curve fitting amounts to finding a compromise between the best fit to the critical magnetic field strengths where the aspect ratio $\left(B_{*}\right)$ or splitcore defect $\left(B_{c}\right)$ starts to grow, and the best fit to the data beyond those critical field strengths. These two choices then produce a value for $\gamma / K_{1}$ and one for $\gamma^{2} / K_{1}$ for the sterically stabilized system and for $w / K_{1}$ and $w^{2} / K_{1}$ for the chargestabilized gibbsite, from which both individual parameter values can be deduced.

The fact that in this way we find two slightly different values for $B_{c}$ despite the fact that $B_{c}$ in Eq. (4) is independent of $\omega$, is caused by the choice of $\aleph=b \gamma / K_{1}$, associated with the core of the line defect. We take it as a constant, which is not exactly true for the different values of $\gamma$ and $K_{1}$ in both fits, but the logarithmic dependence makes the difference in $B_{c}$ quite small. We also remark that even though in both systems we did observe a line defect running throughout the entire tactoid at very high field strengths (as shown in Fig. 3), we omit these last few data points from our curve-fitting procedure. The reason is that including these points would make the general fit to the other points very poor indeed, e.g., with a critical magnetic field strength that almost completely vanishes and, more worryingly, give the fitted curve the wrong curvature. On the other hand and in support of this choice, it is also true that the experimental points at high magnetic field strength have a large degree of uncertainty because the contrast strongly diminishes as the "isotropic" (or rather, paranematic) background also aligns in the magnetic field. By way of consistency check, we use the analytic Eq. (4) for $B_{c}$ in combination with the experimental data of Fig. 3 to derive a value for $K_{1}$ that we compare with the values found from our curve-fitting procedure.

For the curve fitting to the tactoid elongation data that we have obtained for the sterically stabilized system, there is an additional constraint that we require a minimum value of $\omega$ for the observed aspect ratios to occur in the phase diagram. This value turns out to be higher than expected from previous experiments in gibbsite platelets, ${ }^{28}$ which, in fact, is an important observation that we return to later. A value of $\omega=1.5$ turns out not to suffice, but $\omega=2$ does and gives a good fit, so we choose to take this value, which agrees with density functional simulations on hard rectangular platelets. ${ }^{44}$ Here, it must be noted that a value that is, say, $10 \%$ smaller or larger presumably also allows for an acceptable fit.

Finally, as already alluded to in Sec. IV, the emergence of a radial hedgehog director field in the absence of a magnetic field produces a lower bound for the anchoring strength, because this happens according to our theory only if $w$ $>3 K_{1} / R$. Strictly speaking this is true only for the transition from a spherical drop with a uniform field to a radial field; a slightly sharper, i.e., higher, lower bound can be found if we allow for the equilibrium lens shape (shown in Fig. 9). For convenience we use the simple but very accurate result that follows from the spherical drops, so then the choice for $\omega$, and $\Re$ then requires that $\Re \omega>3$. The values for $\omega, \gamma$, and $w$ that follow from these choices and lower bound mentioned above should then all be internally consistent. Because we have observed uniform tactoids in the aqueous gibbsite system, we have yet another check for the obtained values for $K_{1}$ and $w$, because we can independently determine the ratio of $K_{1} / \mathrm{w}$ from the tactoid size where the cross-over from a uniform to a radial director field takes place.

Results of our curve-fitting efforts are discussed in Sec. VI.

\section{ANALYSIS AND DISCUSSION}

Let us first deal with the droplets consisting of the sterically stabilized platelets in bromotoluene, where we rely on the same data for the tactoid elongation as in our previous paper, where we presumed perfect homeotropic surface anchoring. ${ }^{23}$ Hence, we reanalyze these data in the light of our more accurate model described in this work, allowing us to assess the effect of imperfect anchoring on the value of the obtained surface tension, $\gamma$.

We begin with a curve fit of our model to the measured length of the split-core defect (Fig. 3) of a tactoid with an initial radius of $32 \mu \mathrm{m}$ in this system. As we noticed in Sec. V, we omit the data points at high magnetic field strength. The value of $\Re$ that produces the best fit to the critical magnetic field strength turns out to be $\Re=100$, which is rather large, as we will see later. We obtain lower and upper 


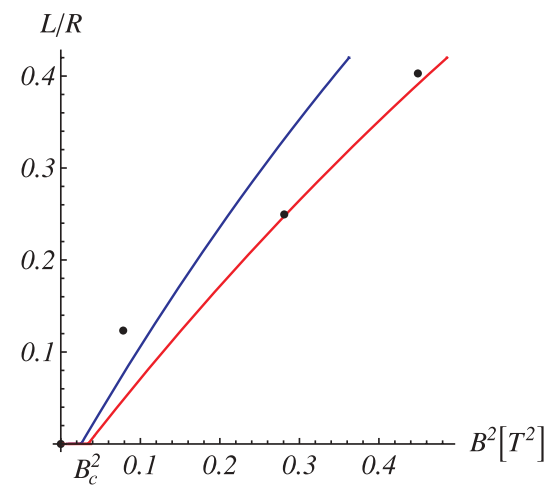

FIG. 12. Ratio of the length of the split-core defect and the radius of the drop, $L / R$, as a function of the square of the magnetic field strength $B^{2}$ for a tactoid in a suspension of sterically stabilized gibbsite in bromotoluene. Tactoid radius: $R=32 \mu \mathrm{m}$. Experimental data: dots; theoretical fits to the data: curves. In order to get good agreement for both the critical magnetic field strength and the ratio $L / R$, we derive a lower and upper bound for $B^{2} / \beta^{2}$ $=\gamma^{2} /\left(-\rho \Delta \chi K_{1}\right)$. We find $B^{2} / \beta^{2}=25 \mathrm{~T}^{2}$ (left curve) and $B^{2} / \beta^{2}=35 \mathrm{~T}^{2}$ (right curve), respectively (see the main text).

bounds for the interfacial tension $\gamma$ and the elastic constant $K_{1}$, of $\gamma=2.1 \cdot 10^{-7} \mathrm{~N} / \mathrm{m}$ and $\gamma=2.8 \cdot 10^{-7} \mathrm{~N} / \mathrm{m}$, and $K_{1}=0.4 \cdot 10^{-13} \mathrm{~N}$ and $K_{1}=0.6 \cdot 10^{-13} \mathrm{~N}$, respectively, with $B_{c}=0.16 \mathrm{~T}$ and $B_{c}=0.18 \mathrm{~T}$ (see Fig. 12). From Eq. (4) we can obtain another value for $K_{1}$ by determining $B_{c}$ from the data for the defect length in Fig. 3. We determine $B_{c}=0.18 \pm 0.2 \mathrm{~T}$, which results in a value for $K_{1}=1.1 \pm 0.5 \cdot 10^{-13} \mathrm{~N}$. These values agree reassuringly well, implying that we believe them to be robust.

Next, we attempt to confirm the previous analysis by a curve fit of our model this time to the aspect ratio of two elongated tactoids with an initial radius of 28 and $31 \mu \mathrm{m}$, respectively, again for the system of sterically stabilized gibbsite. In order to obtain the correct aspect ratio of $a \approx 1.3$ for the tactoids, we have to take $\omega=2$ as described above. The value of $\Re$ that produces the best fit to the critical magnetic field strength is smaller than in our curve fitting to the length of the split-core line defect, because the critical field strength $B_{c}$ needed to elongate a tactoid is larger. For the tactoid of $28 \mu \mathrm{m}$ we take $\Re=25$, and the condition that $\Re \omega>3$ is then also satisfied (see Fig. 13). From the fit we find $B_{*}=0.47 \mathrm{~T}$ and a value of $\gamma=0.9 \cdot 10^{-7} \mathrm{~N} / \mathrm{m}$ with a lower and upper bound of $\gamma=0.6 \cdot 10^{-7} \mathrm{~N} / \mathrm{m}$ and $\gamma=1.2 \cdot 10^{-7} \mathrm{~N} / \mathrm{m}$. The same fit gives $K_{1}=1.0 \cdot 10^{-13} \mathrm{~N}$, with bounds $K_{1}=0.7 \cdot 10^{-13} \mathrm{~N}$ and $K_{1}=1.3 \cdot 10^{-13} \mathrm{~N}$. For the drop with a radius of $31 \mu \mathrm{m}$ we take $\Re=23$ and the best fit then produces $B_{*}=0.30$ $\mathrm{T}$ and values of $\gamma$ and $K_{1}$ of $\gamma=0.4 \cdot 10^{-7} \mathrm{~N} / \mathrm{m}$ with a lower and upper bound of $\gamma=0.2 \cdot 10^{-7} \mathrm{~N} / \mathrm{m}$ and $\gamma=0.7 \cdot 10^{-7} \mathrm{~N} / \mathrm{m}$, and $K_{1}=0.5 \cdot 10^{-13} \mathrm{~N}$ with bounds $K_{1}=0.3 \cdot 10^{-13} \mathrm{~N}$ and $K_{1}=1.0 \cdot 10^{-13} \mathrm{~N}$. Again, these values are quite consistent with those obtained for the smaller drop.

Comparison of the found values for $B_{c}$ (the critical field strength at which the line defect starts to grow) and $B_{*}$ (the critical field strength at which the tactoid elongation starts) shows that $B_{c}<B_{*}$, in agreement with what was found in the theoretical model: upon an increase of the magnetic field first the point defect stretches to a line defect and subsequently at higher magnetic field strength the tactoid is elongated in the field direction. The theory also predicts that the tactoid should become spherical again at even higher magnetic field strength but this was not confirmed in our experiments. It must be noted, though, that at high magnetic field strength a precise measurement of the tactoid properties becomes increasingly difficult, due to alignment of the isotropic background.

All the obtained values for $\gamma$ and $K_{1}$ are summarized in Table I, which also shows the values from the cited earlier work, obtained with a model that assumed complete anchoring. ${ }^{23}$ If we compare the values for $\gamma$ obtained from the tactoid elongation with both models, it is clear that significantly lower values are obtained when incomplete anchoring is taken into account, $(0.4-0.9) \cdot 10^{-7} \mathrm{~N} / \mathrm{m}$ versus $(2-5) \cdot 10^{-7} \mathrm{~N} / \mathrm{m}$, in agreement with the expectation put forward in the previous work. ${ }^{23}$ However, the value we obtained with the new model by the fitting of the length of the line defect as a function of magnetic field strength is somewhat larger, namely $2.5 \pm 0.4 \cdot 10^{-7} \mathrm{~N} / \mathrm{m}$, which results in an average value for the interfacial tension of sterically gibbsite in bromotoluene of $\gamma=1.3 \cdot 10^{-7} \mathrm{~N} / \mathrm{m}$. So, all of the values
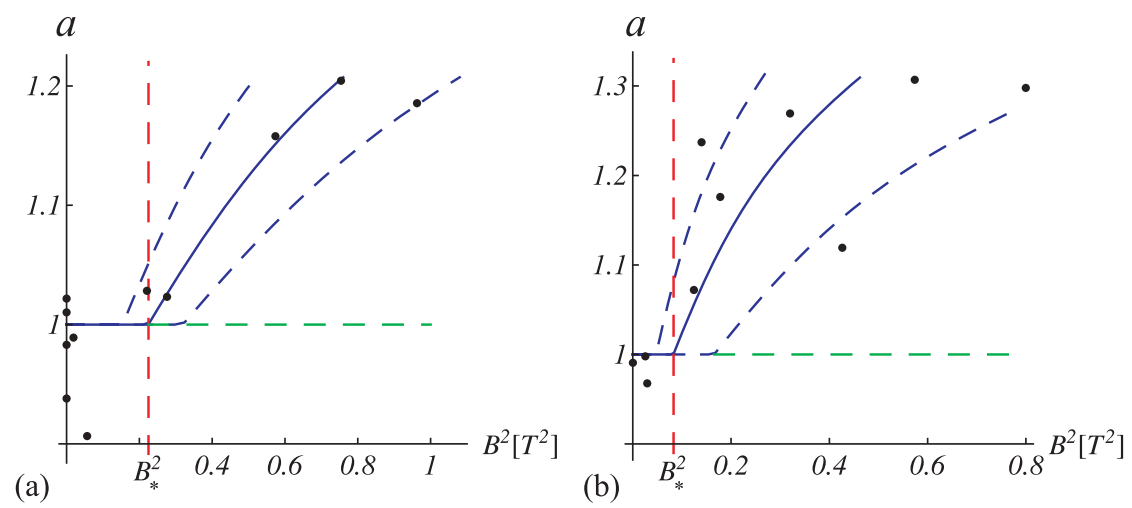

FIG. 13. The aspect ratio $a$ of two tactoids in the sterically stabilized gibbsite dispersion as a function of the square of the magnetic field strength $B^{2}$, where both the critical magnetic field strength $B_{*}$ and the values of $a$ are fitted. The points represent experimental data and the solid lines are fits to our model. For both fits $\omega=2$ has been taken to reach the desired aspect ratios. The fit gives a value for $B^{2} / \beta^{2}=\gamma^{2} /\left(-\rho \Delta \chi K_{1}\right)$ and the dashed lines indicate a lower and upper bound (see the main text). (a) Tactoid with $R=28 \mu \mathrm{m}$ with $B^{2} / \beta^{2}=1.9 \mathrm{~T}^{2}$, giving $\gamma=0.9 \cdot 10^{-7} \mathrm{~N} / \mathrm{m}$ and $K_{1}=1.0 \cdot 10^{-13} \mathrm{~N}$. (b) Tactoid with $R=31 \mu \mathrm{m}$ with $B^{2} / \beta^{2}=0.7 \mathrm{~T}^{2}$ (solid line), giving $\gamma=0.4 \cdot 10^{-7} \mathrm{~N} / \mathrm{m}$ and $K_{1}=0.5 \cdot 10^{-13} \mathrm{~N}$. 


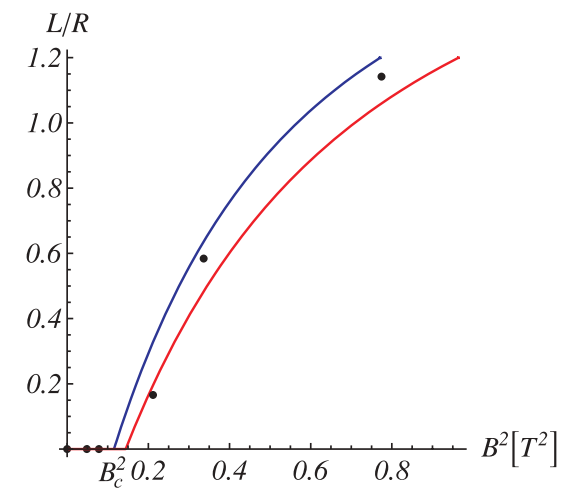

(a)

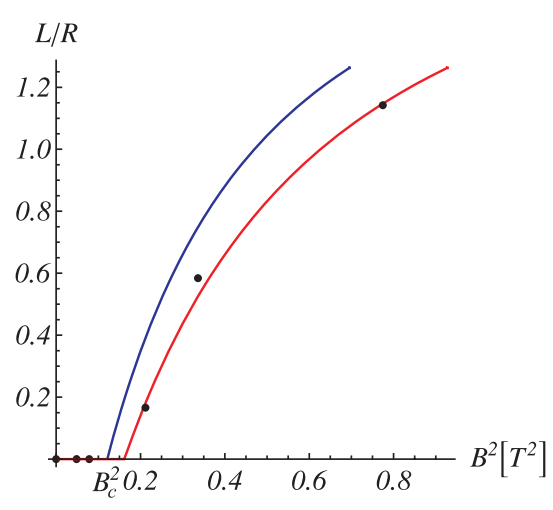

(b)

FIG. 14. Ratio of the length of the split-core defect relative to the tactoid radius, $L / R$, as a function of the square of the magnetic field strength $B^{2}$ for the case of charge-stabilized gibbsite in water. Experimental data: dots, the left and right curves: theoretical curves, optimized with respect to the critical magnetic field strength $B_{c}$ and to the experimental values of $L / R$ at larger field strengths. (a) Tactoid of $R=30 \mu \mathrm{m}$ with $\omega^{2} B^{2} / \beta^{2}=0.4 \mathrm{~T}^{2}$ and $B_{c}=0.34 \mathrm{~T}$ (left curve) and $\omega^{2} B^{2} / \beta^{2}=0.5 \mathrm{~T}^{2}$ and $B_{c}=0.38 \mathrm{~T}$ (right curve). (b) Tactoid of $R=19 \mu \mathrm{m}$ with $\omega^{2} B^{2} / \beta^{2}=0.18 \mathrm{~T}^{2}$ and $\omega^{2} B^{2} / \beta^{2}=0.24 \mathrm{~T}^{2}$, giving $B_{c}=0.35 \mathrm{~T}$ and $B_{c}=0.40 \mathrm{~T}$, respectively (see the main text).

of the surface tension we extract from the tactoid experiments are much larger than the value of $\gamma=3 \cdot 10^{-9} \mathrm{~N} / \mathrm{m}$ obtained from capillary-rise experiments, in the comparable system of sterically stabilized gibbsite in toluene as opposed to bromotoluene. ${ }^{28}$ We come back on some of the drawbacks of our tactoid model below, although we do not believe these drawbacks can actually explain this discrepancy. The values for the elastic splay constant $K_{1}$ of both the fits to the line-defect length and the aspect ratio and $B_{c}$ produce an average of $0.8 \pm 0.3 \cdot 10^{-13} \mathrm{~N}$, which is somewhat smaller than the values that we established in our previous work of $K_{1}=0.9-2.6 \cdot 10^{-13} \mathrm{~N}$, yet still consistent.

Now we turn to the spherical tactoids in dispersions of charge-stabilized gibbsite in water, tactoids that do not deform even for the highest magnetic field strengths tested. As explained in Sec. V, the fitting procedure for the length of the split-core defect in the charge-stabilized system is equal to that in the sterically stabilized system, apart from the fact that $\gamma$ is now replaced by $w$. We have to find the value of $\Re \omega$ that gives the best agreement with the critical magnetic field strength $B_{c}$, where we again disregard the points at very high field strengths where $L / R \approx 2$. The fits give a lower and upper bound for $\omega^{2} B^{2} / \beta^{2}=w^{2} /\left(-\rho \Delta \chi K_{1}\right)$.
We use the measured length of the split-core defect of two tactoids of radius 30 and $19 \mu \mathrm{m}$, respectively, as depicted in Fig. 3. For the drop with $R=30 \mu \mathrm{m}$ we take the value $\Re \omega=10$, and the fits with the best curvature and the best fit to the data give a lower and upper bound for $w$ of $w=4.8 \cdot 10^{-8} \mathrm{~N} / \mathrm{m}$ and $w=6.0 \cdot 10^{-8} \mathrm{~N} / \mathrm{m}$, and $K_{1}$ between $1.4 \cdot 10^{-13} \mathrm{~N}$ and $1.8 \cdot 10^{-13} \mathrm{~N}$, where the critical magnetic field strengths vary between $B_{c}=0.34 \mathrm{~T}$ and $B_{c}$ $=0.38 \mathrm{~T}$ (see Fig. 14). If we use Eq. (4) with the data in Fig. 3 we find $B_{c}=0.34 \pm 0.2 \mathrm{~T}$, which results in a value for $K_{1}$ $=3.5 \pm 0.4 \cdot 10^{-13} \mathrm{~N}$. For the smaller drop with $R=19 \mu \mathrm{m}$ we take the proportional value $\Re \omega=6.3$, and we find from the best fit to the data bounds of $w$ of $w=2.2 \cdot 10^{-8} \mathrm{~N} / \mathrm{m}$ and $w=2.9 \cdot 10^{-8} \mathrm{~N} / \mathrm{m}$, and $K_{1}$ between $6.5 \cdot 10^{-14} \mathrm{~N}$ and $8.7 \cdot 10^{-14} \mathrm{~N}$, and $B_{c}$ between $0.35 \mathrm{~T}$ and $0.40 \mathrm{~T}$. From Eq. (4) and Fig. 3 we find $B_{c}=0.32 \pm 0.2 \mathrm{~T}$ and $K_{1}=1.4 \pm 0.2$ $\cdot 10^{-13} \mathrm{~N}$. These values for $K_{1}$ are again reasonably consistent with each other.

The values for the elastic constant $K_{1}$ and the anchoring strength $w$ in aqueous gibbsite that we obtained from the fits of the theoretical model to the experimental data are summarized in Table II. If we average the obtained values, we find for $K_{1}$ a value of $2 \pm 1 \cdot 10^{-13} \mathrm{~N}$ and for $w$ a value of

TABLE I. Results of the fitting procedure for sterically stabilized gibbsite in bromotoluene. The first three columns of the table give values for $K_{1}$ from Eq. (4) for $B_{c}$ and average values for $K_{1}$ and $\gamma$ that follow from the upper and lower bound fits of the model to the relative split-core-defect length $L / R$, as depicted in Fig. 12. The next two columns give the best fit values for for $K_{1}$ and $\gamma$ that follow from the fits of the model to the elongation of the tactoid, i.e., the aspect ratio $a$, as depicted in Fig. 13. The last column presents results obtained with the old model involving complete anchoring $(\omega \rightarrow \infty)$.

\begin{tabular}{|c|c|c|c|c|c|c|}
\hline & \multicolumn{5}{|c|}{$\omega=2$} & \multirow{3}{*}{$\begin{array}{c}\omega \rightarrow \infty \\
\text { Elongation } \\
\gamma\left[10^{-7} \mathrm{~N} / \mathrm{m}\right.\end{array}$} \\
\hline & \multicolumn{3}{|c|}{ Line defect } & \multicolumn{2}{|c|}{ Elongation } & \\
\hline & $K_{1}\left[10^{-13} \mathrm{~N}\right]$ & $K_{1}\left[10^{-13} \mathrm{~N}\right]$ & $\gamma\left[10^{-7} \mathrm{~N} / \mathrm{m}\right]$ & $K_{1}\left[10^{-13} \mathrm{~N}\right]$ & $\gamma\left[10^{-7} \mathrm{~N} / \mathrm{m}\right]$ & \\
\hline & $B_{c}$ & \multicolumn{2}{|c|}{ Fit $L / R$} & \multicolumn{2}{|c|}{ Fit $a$} & Fit $a$ \\
\hline$R=32 \mu \mathrm{m}$ & $1.1 \pm 0.5$ & $0.5 \pm 0.1$ & $2.5 \pm 0.4$ & - & - & - \\
\hline$R=28 \mu \mathrm{m}$ & - & - & - & $1.0 \pm 0.3$ & $0.9 \pm 0.3$ & $5 \pm 2$ \\
\hline$R=31 \mu \mathrm{m}$ & - & - & - & $0.5 \pm 0.5^{\mathrm{a}}$ & $0.4 \pm 0.3$ & $3 \pm 2$ \\
\hline
\end{tabular}

${ }^{a}$ This rather large value of the error is a consequence of the fact that the upper and lower bounds are asymmetrically deviating from the best fit, thus the lower bound is in fact not zero, but $0.3 \cdot 10^{-13} \mathrm{~N}$. 
TABLE II. Values for the splay elastic constant $K_{1}$ and the anchoring strength $w$ of the charge-stabilized gibbsite, obtained by fitting our theory to the experimental data for droplets of radius 30 and $19 \mu \mathrm{m}$. Values for $K_{1}$ are obtained individually from the critical magnetic field strength, $B_{c}$, and together with values for $w$ from fit to the magnetic-field dependence of the dimensionless length of the line defect $L / R$ in Fig. 14, i.e., from the choice of $\Re$ as well as from the curvature fits. The values from the fits of $L / R$ are averaged over the values obtained from the upper and lower bound fits. See also the main text.

\begin{tabular}{lcccc}
\hline \hline & \multicolumn{2}{c}{$K_{1}\left[10^{-13} \mathrm{~N}\right]$} & & $\frac{w\left[10^{-8} \mathrm{~N} / \mathrm{m}\right]}{$\cline { 2 - 3 }} \\
\cline { 2 - 3 } & $B_{c}$ & Fit $L / R$ & & Fit $L / R$ \\
\hline Tactoid $R=30 \mu \mathrm{m}$ & $3.5 \pm 0.4$ & $1.6 \pm 0.2$ & & $5.4 \pm 0.6$ \\
Tactoid $R=19 \mu \mathrm{m}$ & $1.4 \pm 0.2$ & $0.8 \pm 0.1$ & & $2.5 \pm 0.4$ \\
\hline \hline
\end{tabular}

$4 \pm 2 \cdot 10^{-8} \mathrm{~N} / \mathrm{m}$. As explained in Sec. V, we can also independently determine a value for the ratio of $K_{1} / w$ from the cross-over tactoid size of the transition from a uniform to a radial director field with increasing tactoid size. We have observed tactoids with a uniform director field with size up to about $40 \mu \mathrm{m}$, while the smallest tactoid with a radial director field measured $38 \mu \mathrm{m}$ in diameter. As the cross-over tactoid radius is determined by $R=3 K_{1} / w$, this provides us with a value of $K_{1} / w$ of $6.5 \mu \mathrm{m}$. The value for $K_{1} / w$ that follows from our curve fitting is $5 \mu \mathrm{m}$, which is surprisingly close. It is, we repeat, not possible to extract a value for $\gamma$ in aqueous gibbsite suspensions because the tactoids remain spherical, at least to within the measurement error. What we can say, though, is that $\omega$ must be at least smaller than 1 and $\gamma$ therefore larger than $4 \cdot 10^{-8} \mathrm{~N} / \mathrm{m}$, because if this would not have been the case, it would have led to a tactoid elongation of $10 \%$, which should have been perceptible in the experiments.

It is of interest to compare the anchoring strengths for the two cases discussed in this paper, i.e., tactoids in sterically and charge-stabilized dispersions of gibbsite of very comparable dimensions. For the sterically stabilized gibbsite system, we can calculate the anchoring strength from the definition $\omega \equiv w / \gamma$. We use the value of $\omega=2$ that we obtained to be able to get a good fit to the experimental data on the magnetic-field dependence of the aspect ratio of an elongated tactoid. For $\gamma$ we take an average of what we found from the various fitting procedures, $\gamma=1.3 \cdot 10^{-7} \mathrm{~N} / \mathrm{m}$, resulting in a value for the anchoring strength of $w=2.6 \cdot 10^{-7} \mathrm{~N} / \mathrm{m}$. This is almost one order of magnitude larger than the anchoring strength of the charge-stabilized gibbsite system in water ( $w=4 \cdot 10^{-8} \mathrm{~N} / \mathrm{m}$ ). It is not clear what the origin is of this difference, where we note that other properties, such as the splay-elastic constant $K_{1}$, are quite similar for the two types of system. The values we find for $K_{1}, 0.8 \pm 0.3 \cdot 10^{-13} \mathrm{~N}$ for sterically stabilized gibbsite and $2 \pm 1 \cdot 10^{-13} \mathrm{~N}$ for aqueous gibbsite, are in good agreement with computer simulations. ${ }^{45}$ Furthermore, they are about a factor of 2 larger than was previously found by Van der Beek et al. ${ }^{39}$ for the bend-elastic constant $K_{3}$ in the comparable system of sterically stabilized gibbsite in toluene, which qualitatively agrees with theoretical predictions. $^{46}$

We end this section by a brief discussion of the caveats of the theoretical model that we advance in this paper. For instance, we assume that all the model parameters, including the splay-elastic constant $K_{1}$, the anchoring strength $w$, and the surface tension $\gamma$ are independent of the magnetic-field strength. However, with increasing magnetic field strength the order parameter $S$ of the nematic phase increases, which plausibly will also affect $K_{1}$ and $w$. While elastic constants are generally accepted to be proportional to the square of the nematic order parameter, $S^{2}$, the anchoring strength is linear in this order parameter. ${ }^{38}$ Because both the elastic constant and the anchoring strength counterbalance the deformation by the magnetic field, an underestimation of $K_{1}$ could lead to a value too high for the anchoring strength and interfacial tension. Furthermore, the anchoring strength might also depend on the magnetic field strength due to alignment of the isotropic phase in the field. However, correction for the latter would lead to an even higher value for the anchoring strength in zero field.

A contribution that is missing in our free-energy analysis is bend deformation, which might be present at high magnetic field where the line defect spans the entire tactoid. Although difficult to infer from the polarized light microscopy experiments, the curvature in the dark horizontal band (Fig. 2) might point in that direction. Indeed, for plate-like particles, the bend-elastic constant is thought to be smaller than the splay constant, ${ }^{45}$ so some elastic energy can be gained. An appropriate bend deformation also makes the anchoring less unfavorable, producing an additional reduction of the free energy. At this point, it is difficult to judge in what way that this should influence the results of our curve fitting.

In any event, it seems quite unlikely that these caveats could be responsible for a difference of almost two orders of magnitude between the interfacial tension that we find here and that obtained from capillary rise experiments. The origin of this discrepancy remains a mystery. We intend to discuss in a forthcoming publication a detailed investigation of tactoid properties as a function of tactoid size in both gibbsite systems that results in comparable values for $K_{1} / w$ that we find here. ${ }^{35}$

\section{CONCLUSION}

We find that nematic tactoids that form in dispersions of plate-like colloids exhibit an interesting response to an externally applied magnetic field due to the homeotropic surface anchoring of the platelets. As the director-field configuration is a function of the tactoid size, we have been able to observe a number of transitions between different kinds of director-field structure and droplet shape with increasing magnetic field strength. Perhaps the most remarkable observation is that large tactoids that tend to be spherical and have a radial director field emanating from a hedgehog point defect in their center develop a split-core defect in a sufficiently strong field. The split-core defect develops only provided the diamagnetic susceptibility anisotropy is negative - a prediction made ten years ago by Mkaddem and Gartland ${ }^{27}$ that we have been able to confirm.

We focused our experiments on two types of system: charged gibbsite dispersed in water and sterically stabilized gibbsite in bromotoluene. We found these systems to behave very differently. In a magnetic field the latter not only develop the the split-core defect structure but also become elongated 
in the field direction. This is not so for the former, these remain spherical. The difference in behavior of the two types of dispersion is caused by the relative magnitude of the anchoring strength and the surface tension, which for the former is small and for the latter large.

We have been able to account for the observed transitions by invoking a macroscopic description that balances elastic, surface, and magnetic free energies and predicts the structure of the tactoids as a function of their size, the splay elastic constant, surface tension and anchoring strength, and the magnetic field strength. Our idealized theory allowed us to extract values for the splay-elastic constant $\left(K_{1}\right)$, interfacial tension $(\gamma)$, and anchoring strength $(w)$ from our observations. Not surprisingly, we found remarkable differences between the sterically stabilized gibbsite in bromotoluene and the aqueous gibbsite system.

The anchoring strength is much larger in the sterically stabilized system, with a dimensionless anchoring strength $\omega$ of about 2, while that of the charge-stabilized system must approach zero, implying that they represent the strong and weak anchoring limit respectively. The values for the splay elastic constant $K_{1}$ for the two systems are actually quite close, varying by no more than a factor of two. The value for the surface tension $\gamma$ that we find here for the sterically stabilized gibbsite is still larger by two orders of magnitude than was previously found from capillary rise experiments, ${ }^{28}$ a discrepancy for which we have no explanation.

\section{ACKNOWLEDGMENTS}

The authors are grateful to B.W.M. Kuipers for constructing the polarized light microscope setup in magnetic field. A.A.V. would like to thank the Royal Netherlands Academy of Arts and Sciences (KNAW) for financial support. The work of R.H.J.O. forms part of the research programme of the Dutch Polymer Institute (DPI, project 648).

${ }^{1}$ R. D. Williams, Rutherford Appleton Laboratory Report No. RAL-85-028 (1985) (unpublished).

${ }^{2}$ P. S. Drzaic, Liquid Crystal Dispersions (World Scientific Publishing Co. Pte. Ltd., Singapore, 1995).

${ }^{3}$ O. D. Lavrentovich, Liq. Cryst. 24, 117 (1998).

${ }^{4}$ H. Zocher, Anorg. Chem. 147, 91 (1925).

${ }^{5}$ A. V. Kaznacheev, M. M. Bogdanov, and S. A. Taraskin, J. Exp. Theor. Phys. 95, 57 (2002).

${ }^{6}$ J. D. Bernal and I. Fankuchen, J. Gen. Physiology 25, 111 (1941).

${ }^{7}$ Z. Dogic and S. Fraden, Phil. Trans. R. Soc. A 359, 997 (2001).

${ }^{8}$ P. W. Oakes, J. Viamontes, and J. X. Tang, Phys. Rev. E 75, 061902 (2007).
${ }^{9}$ Y. Trukhina, S. Jungblut, P. van der Schoot, and T. Schilling, J. Chem. Phys. 130, 164513 (2009).

${ }^{10}$ A. Cuetos and M. Dijkstra, Phys. Rev. Lett. 98, 095701 (2007).

${ }^{11}$ E. Berggren, C. Zannoni, C. Chiccoli, P. Pasini, and F. Semeria, Chem. Phys. Lett. 197, 224 (1992).

${ }^{12}$ M. A. Bates, Chem. Phys. Lett. 368, 87 (2003).

${ }^{13}$ A. V. Kaznacheev, M. M. Bogdanov, and A. S. Sonin, J. Exp. Theor. Phys. 97, 1159 (2003)

${ }^{14}$ P. Prinsen and P. van der Schoot, Phys. Rev. E 68, 021701 (2003).

${ }^{15}$ P. Prinsen and P. van der Schoot, Eur. Phys. J. E 13, 35 (2004).

${ }^{16} \mathrm{Z}$. Dogic (unpublished).

${ }^{17}$ K. Shundyak and R. van Roij, J. Phys.: Condens. Matter 13, 4789 (2001).

${ }^{18}$ P. van der Schoot, J. Phys. Chem. B 103, 8804 (1999).

${ }^{19}$ Z. Y. Chen and J. Noolandi, Phys. Rev. A 45, 2389 (1992).

${ }^{20}$ S. M. Cui, O. Akcakir, and Z. Y. Chen, Phys. Rev. E 51, 4548 (1995).

${ }^{21}$ D. L. Koch and O. G. Harlen, Macromolecules 32, 219 (1999).

${ }^{22}$ R. H. J. Otten and P. van der Schoot, Langmuir 25, 2427 (2009).

${ }^{23}$ A. A. Verhoeff, R. H. J. Otten, P. van der Schoot, and H. N. W. Lekkerkerker, J. Phys. Chem. B 113, 3704 (2009).

${ }^{24}$ P. Davidson, private communication (2010).

${ }^{25}$ E. Dubois-Violette and O. Parodi, J. Physique Coll. 4, 57 (1969).

${ }^{26}$ S. Kralj and S. Zumer, Phys. Rev. A 45, 2461 (1992).

${ }^{27}$ S. Mkaddem and E. C. Gartland, Phys. Rev. E 62, 6694 (2000).

${ }^{28}$ D. van der Beek, H. Reich, P. van der Schoot, M. Dijkstra, T. Schilling, R. Vink, M. Schmidt, R. van Roij, and H. Lekkerkerker, Phys. Rev. Lett. 97, 087801 (2006).

${ }^{29}$ A. M. Wierenga, T. A. J. Lenstra, and A. P. Philipse, Colloids Surf. A 134, 359 (1998)

${ }^{30}$ P. A. Buining and H. N. W. Lekkerkerker, J. Phys. Chem. 97, 11510 (1993).

${ }^{31}$ F. M. van der Kooij and H. N. W. Lekkerkerker, J. Phys. Chem. B 102, 7829 (1998).

${ }^{32}$ M. P. B. van Bruggen, M. Donker, H. N. W. Lekkerkerker, and T. L. Hughes, Colloids Surf. A 150, 115 (1999).

${ }^{33}$ D. van der Beek, A. V. Petukhov, P. Davidson, J. Ferre, J. P. Jamet, H. H. Wensink, G. J. Vroege, W. Bras, and H. N. W. Lekkerkerker, Phys. Rev. E 73, 041402 (2006)

${ }^{34}$ A. A. Verhoeff, R. P. Brand, and H. N. W. Lekkerkerker, "Tuning the birefringence of the nematic phase in suspensions of colloidal gibbsite platelets," Mol. Phys. (submitted).

${ }^{35}$ A. A. Verhoeff, I. A. Bakelaar, R. H. J. Otten, P. van der Schoot, and H. N. W. Lekkerkerker, Langmuir 27, 116 (2011).

${ }^{36}$ A. Fernandez-Nieves, D. R. Link, M. Marquez, and D. A. Weitz, Phys. Rev. Lett. 98, 087801 (2007).

${ }^{37}$ A. Rapini and M. J. Papoular, J. Physique Coll. 30, 54 (1969).

${ }^{38}$ P. G. de Gennes, The Physics of Liquid Crystals (Oxford University Press, New York, 1974).

${ }^{39}$ D. van der Beek, P. Davidson, H. H. Wensink, G. J. Vroege, and H. N. W. Lekkerkerker, Phys. Rev. E 77, 031708 (2008).

${ }^{40}$ G. Wulff, Z. Kristallogr. 34, 449 (1901).

${ }^{41}$ R. H. J. Otten and P. van der Schoot, "Nematic droplets of plate-like particles in a magnetic field" (to be published).

${ }^{42}$ R. B. Zasadzinski and J. Meyer, Phys. Rev. Lett. 56, 636 (1986).

${ }^{43}$ W. Song, I. A. Kinloch, and A. H. Windle, Science 302, 1363 (2003).

${ }^{44}$ M. Bier, L. Harnau, and S. Dietrich, Phys. Rev. E 69, 021506 (2004).

${ }^{45}$ P. A. O'Brien, M. P. Allen, D. L. Cheung, M. Dennison, and A. Masters, Phys. Rev. E 78, 051705 (2008).

${ }^{46}$ M. A. Osipov and S. Hess, Mol. Phys. 78, 1191 (1993). 